\title{
On Two-Tier Femtocell over Fading Environments
}

\author{
Joy Iong-Zong Chen, Kai-Chih Chuang, Ching-Chuan Chiu, Deng-Jyi Juang \\ Department of Electrical Engineering, Dayeh University \\ Changhwa, Taiwan \\ E-mail: jchen@mail.dyu.edu.tw
}

Received December 18, 2010; revised March 3, 2011; accepted March 16, 2011

\begin{abstract}
In this paper the system performance of an MC-CDMA (multi-carrier coded-division multiple-access) system operating over single-cell with two-tier femtocell environment is analyzed. Consider two-tier scenario is deployed with a macrocell site in which is being surrounded several femtocells, which are designed to serve a group of subscribers locate in a small coverage area such as small office, home office or a house. The coverage area is typically up to 100 meters in radius. Mostly, the femtocell is applied to serve indoor subscribers, thus, the Rayleigh fading is adopted to characterize the propagation channel. The technique of TH-MCCDMA (time-hopped multi-carrier coded-division multiple-access) technique is supposed to transmit each symbol alternatively with fair time (slotting)/frequency (coding) for each user in the hotspots (the area around $0^{\text {th }}$ femtocell). The intensity of signals estimated at a mobile unit located in the second tie, i.e., the femtocell coverage area, is an important issue. The contribution of the paper is mainly to evaluate the system performance with both the BER (bit error rate) and OP (outage probability) according to the most important parameters, for example, the activating user number, the hopping number provided by TH-MC-CDMA system and the subcarrier number. Furthermore, the discussion of interference avoidance is also discussed with non-analytically.
\end{abstract}

Keywords: Time-Hopped CDMA (TH-CDMA), Hotspot, Femtocell, Macrocell, MC-CDMA, Rayleigh Fading

\section{Introduction}

Recently, the last mile transmission has attracted a huge of attention due to the fact that cell phones have become indispensable in people's life, especially, in indoor wireless transmission. In order to go through the full path that provides enough intensity of signal to arrive at the mobile unit of a subscriber located at indoor site where is a small coverage area, such as a building, home office, small firm or even in a mobile vehicle. The femtocell with two-tier network is being installed to improve the cellular capacity. The reasons for a significant interest has focused in recent on the supplication of femtocells are: it is defined widely in the telecommunications industry as easily installed, low-cost, and low power cellular BS (base station) that works in licensed spectrum to link traditionally to a mobile operation network without modifying mobile terminals. In general, the co-sited users (i.e., the mobile users located under the same coverage of a femtocell) are limited due to the coverage is smaller than other kinds of base station (BS). On contrast, the less interference caused by the other subscribes can serve higher reuse of spectrum. Moreover, decentralized strategies for interference management may be much suitable for femtocells, because of femtocells are mainly costing in traffic, installation fee, and without any operator influence [1].

Specifically, it is known that there are two alternate technologies for deploying femtocells coverage, i.e., relay and WiFi (IEEE802.16j). The coverage of radius for a femtocell is in tens of meters, and it is much smaller than that of the macrocells radius range about in hundreds of meters. Except the advantages mentioned before, the others are including prolong handset battery life and obtain a higher SINR (signal-to-interference-plus-noise ratio) [2]. However, the more are the desired signal components, the less the interference plus noise components are in a wireless system usually. Up to this moment, since the macrocell has a much larger communication radius than that of a femtocell, and the centralized deployment of microcell site is adopted generally, the methods of loading balance user were proposed in [3], in 
which the authors derived by using load balancing metrics to optimize the hierarchical wireless data networks. The research published in [4] has analyzed an operation planned underlay of a macrocell with single/multiple microcells. The focus of outage probability analysis accounting for shadowing effects, cellular geometry, and cross-tier CCI (co-channel interference) are presented in [5]. In addition, an robust interference avoidance scheme is also proposed to enable two-tier networks with universal frequency reuse to obtain higher channel capacity. By proposing the related issues to improve coverage by regulating femtocell transmit power is researched in [6]. The maximum femtocell contention densities at a distance between macrocell BS and analytically to search the solution of coverage zones within subscribers can arrive at the QoS (quality of service) requirement are proposed in [7]. It is well known that the cell site plan is one of the impact factors for system performance of a user under a two-tier femtocell environment. In [8] the authors studied in tiered networks have definitely assumed that an operator planned underlay of a macrocell with single/multiple microcells. The investigation in CR (cognitive radio) with the schemes of sensing-throughput tradeoff for computing optimal sensing time by CR users and limit transmit power of CR users are presented in [9] and $[10,11]$, respectively. The analysis of channel capacities in an ad hoc network with spatial diversity is illustrated in [12]. Though the factor has been proof that nearly 25 multiplies improvement in overall spatial reuse and has been proposed in related researches in [12], in which the assumption is moving from a marcocell-only network to a two-tier underlay with 50 femtocells per cell-site with the OP (outage probability) criteria. The research of system performance for time-hopping MCCDMA (multi-carrier coded-division multiple-access) system has been reported in [13], however, it didn't include the discussion of two-tier femtocell environments. On the basis of inspecting the review to the results from aforementioned published paper, it is known that to analytically evaluate the system performance of an MCCDMA system considered located on the environment with two-tier femtocell network is sparse. It motivates this paper to deploy a scenario of modeling with time and spatial dynamic of users in a wireless radio system to review partly the theory of the Poisson process for an two tier femtocell applications, and to propose the evaluation of system performance for an MC-CDMA system experienced in fading channel under an two-tier femtocell network. It is also a roadmap proposed by [14] for interference avoidance purpose. By figuring out the skeleton of the interference in the case assumes that the femtocell BS and users are randomly distributed in a macrocell, named as a Spatial Poisson Point Process
(SPPP) [15], the BER (bit error rate) and OP can be determined at a femtocell base station of interest. On the other hand, in this paper the system performance with BER and OP expressions is investigated to concentrate attention on considering some possibly caused interferences that a referenced user suffering from the surrounding real world transmitting environments due to a two-tier femtocell application.

Seriously speaking, in order to obtain the most completely analytical results of the system performance for mobile unit operating in such an deployed environment with short-term fading, at least three terms of the RF (radio frequency) interferences should be involved, for instance, (A) macrocell-to-femtocell interference, (B) femtocell-to-femtocell interference, (C) femtocell-tomacrocell interference, arising from a femtocell-based cellular should be included in the analysis of system performance for an MC-CDMA system. Apart from, the three terms pointed out previously, the really involved interferences which are necessary for the evaluation of system performance should be depending on the propagation channel models and the multiple access techniques, e.g., TH-CDMA, TH-MC-CDMA, or TH-OFDMA, etc.. Such that the fast time scale fading channel is not appropriate for indoor wireless communications, nevertheless the multipath effects needs to take into account an indoor propagation model. This report is organized as follows. In Sect. II, the channel environments of an two-tier femtocell network are analyzed first; then the statistical analysis of the MC-CDMA system is derived in Section 3 , following the analytical results reported in Section 4, a brief conclusion is drawn in Section 5.

\section{Description of Channel Environments}

Under the condition which assumes that a two-tier femtocell structure is shown in Figure 1. There are $M$ femtocells, in which is with $K$ users normally distributed, in a single macrocell site. An MC-CDMA system is adopted as the transmission technique and TH-CDMA (time-hopped CDMA) scheme is applied to dominate the time-slot (multiplex) for all the $M$ femtocells included in macrocell. In reviewing the spatial Poisson traffic, the story of categorizing that the interferences occur in the deployment of two-tier femtocell environment should be described first with the view of the Poisson processed point [15]. Consider the subscribes are modeled as a random with spatial Poisson pattern on an sizable space $\varepsilon \in \mathbb{R}^{2}$, which is seen as a space for deploying the macrocell with radius $R_{\text {mac }}$, and the mobile units are locating in space $\varepsilon$ with deterministic location. The uplink MC-CDMA system mobile links to a cellsite is specifically focused on the statistical characteristics estimated 
Macro-cell BS 獣 Tier 1 Macrocell user Femto-cell BS 2 Femtocell user

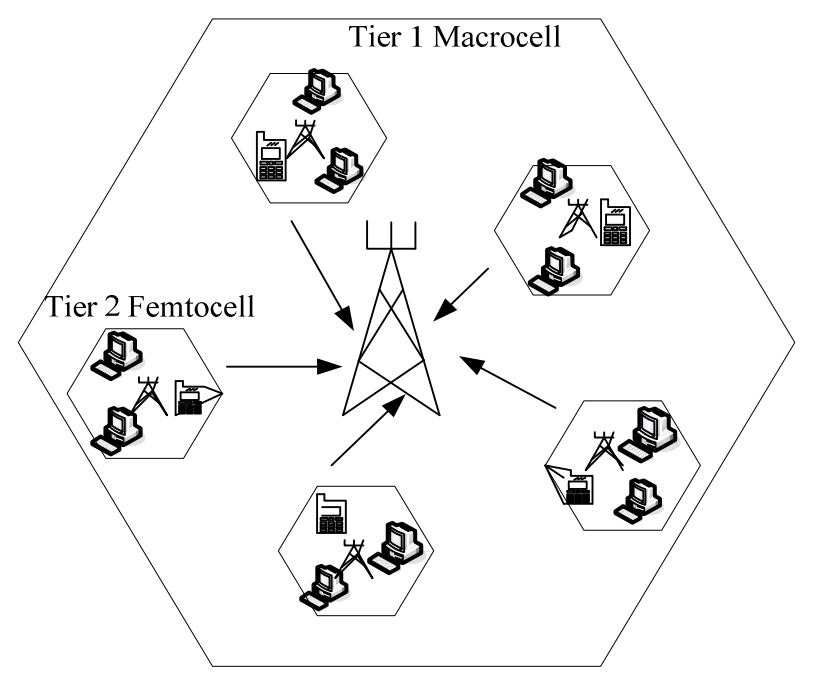

Figure 1. The configuration of an two-tier femtocell application.

at same assigned space within space $\varepsilon$.

Lemma 1. As a Poisson process with expectation value, $m_{p}$, happened in the sizable space $\varepsilon$, the process is a random subset $\Pi$ in $\varepsilon$, thus the following facts are satisfied,

1) The random subset $\Pi$ acts as a femtocell with radius $R_{f e m}$ and a sizable point pattern.

2) $N_{\Pi}^{X}$ denotes the number of points, see it as the number of mobile users within a femtocell, located in $\Pi$ that lies in area $X$, where for $X \subseteq \varepsilon$.

3) $N_{\Pi}^{X}$ and $N_{\Pi}^{Y}$ are generally considered independent each other for all non-contiguous subset $X$, $Y \subseteq \varepsilon$.

Thus, the determination of the total mean and variance of the interference, $f(X)$, where $X \in \Pi$, in the case of assuming that the femtocell BS and users are randomly distributed in a microcell, i.e., in a SPPP area, they can adopt that the expression given as [16]

$$
E\left(\sum_{X \in \Pi} f(X)\right)=\lambda \int_{\mathbb{R}^{2}} f(x) \mathrm{d} x
$$

and

$$
\operatorname{var}\left(\sum_{X \in \Pi} f(X)\right)=\lambda \int_{\mathbb{R}^{2}} f^{2}(x) \mathrm{d} x
$$

respectively, where $\lambda$ is the intensity of $X$, i.e., the mean number of points per unit space. Finally, the different kinds of moment of interference exist in a two tier femtocell channel environment can be obtained by using of the aforementioned procedures.
Accordingly, for the purpose of calculating the BER performance for a referenced subscriber operating in an two-tier femtocell system, the MRC (maximal ratio combining) diversity scheme is assumed to implement for receiving the signal. Thus, to determine the SINR (signal-to-interference-plus-noise) at the output of the MRC for the referenced user is necessary, and it is written as

$$
\Upsilon_{S I N R}=\frac{\left(S_{F E M}\right)^{2}}{\sigma_{I_{T}}^{2}}
$$

where $S_{F E M}=E\left[S_{f e m}\right]$ is the average value of the received signal for the referenced user, it can be obtained as

$$
S_{F E M}=\sqrt{\frac{P_{r, F}}{2 N}} \cdot E\left[\sum_{n=0}^{N-1}\left(\beta_{n, 0}\right)^{2}\right]
$$

where $P_{r, F}$ denotes the received power of a user within a femtocell, $N$ is the number of subcarrier, $\beta_{n, 0}$ indicates the channel fading fraction of the 0 th received path at a femtocell site. The total interferences, $\sigma_{I_{T}}^{2}$, shown in the denominator of (3) can be expressed as $\sigma_{I_{T}}^{2}=\sigma_{\text {CO-Tier }}^{2}+\sigma_{\text {Cross-Tier }}^{2}+\sigma_{I_{A W G N}}^{2}$, where, the first term, $\sigma_{\text {CO-Tier }}^{2}$, denotes as all the components of interference caused from the sources of same tier around the femtocell, and it is named as co-tier interference. The second term, $\sigma_{\text {Cross-Tier }}^{2}$, is designated as all the components of interferences come from the sources of different tier around the femtocell, and it is called as cross-tier interference. These two kinds of interference would be analyzed later. While the last term, $\sigma_{I_{A W G N}}^{2}$, expresses the AWGN component with a double-sided power spectral density of $N_{0} / 2$, which is able to be calculated as

$$
\sigma_{I_{A W G N}}^{2}=\frac{N_{0}}{4 T_{b}} \sum_{n=0}^{N-1}\left(\beta_{n, o}\right)^{2}
$$

where bit interval is expressed as $T_{b}$ which is assumed smaller than the TH-MC-CDMA period, that is, $\mathrm{T}_{b} \ll T=N \cdot T_{c}$, where $N$ denotes the processing gain, and $T_{c}$ is the chip-time of the PN (pseudo-noise) sequence for a TH-MC-CDMA system.

Furthermore, interferences belong to co-tier and crosstier interference can be explained by the diagram shown in Figure 2 with the originality. A deployment with two tier femtocell example, where there are 5 macrocells working in the first tier is considered. With the expression of $M_{0}, \cdots, M_{4}$, in which $M_{0}$ is chosen as the referenced macrocell, and the active user's numbers for each macrocell is assumed as $U_{M_{0}}, \cdots, U_{M_{4}}$. On the other hand, the femtocells are overlapped within the first tier and the referenced femtocell is considered as $F_{M_{0}, 0}$, then the other femtocell belongs to the macrocell of the 


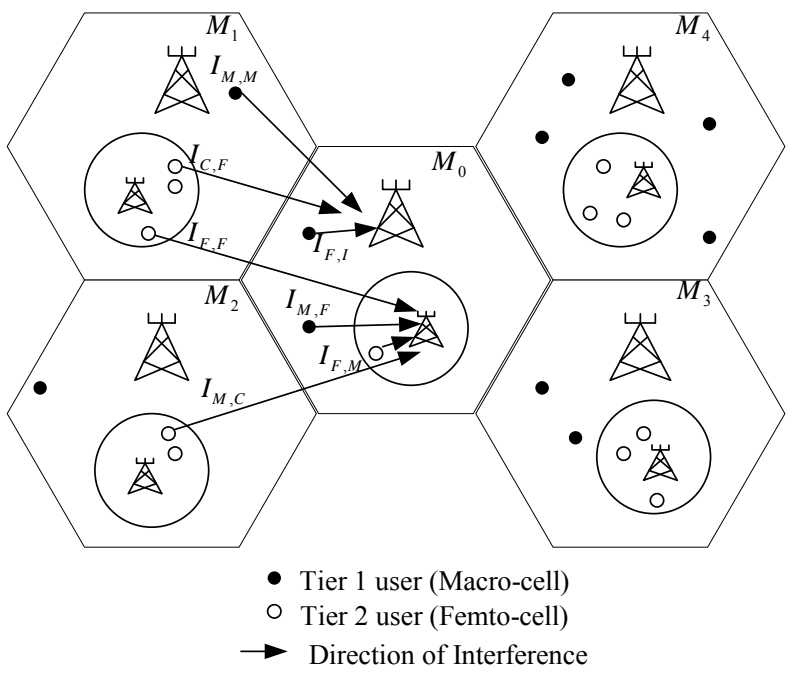

Figure 2. The diagram shows different kind of interferences occur in a two-tier femtocell.

first tier is indexed as $F_{M_{1}}, \cdots, F_{M_{4}}$. Similarly, $U_{F_{M_{1}}}, \cdots, U_{F_{M_{4}}}$ are used to express the active subscribers number located on the second femtocell. The figure says that the subscriber, $U_{M_{0}}$, in macrocell occupies the first time slot is communicating with the macrocell $M_{0}$. The femtocell $F_{M_{0}, 0}$ holds on the second time slot, and femtocell $F_{M_{0}, 1}$ is within the third time slot, and so on.. Moreover, the interferences structure can be described by the Figure 3, it is naturally known that the interferences are comprised by two main parts, which are including the interference comes from the macrocell (co-tier interference), the other is the interference comes from different femtocells (cross-tier interference). However, for the reasons of simplicity in analytically analyze and without loss the generality, it is only the interference mentioned below will be included in this report: the interference comes from different femtocell is with $I_{F, I}$; the interference comes from same femtocell is with $I_{F, F}$; the interference induces from the macro-cell is with $I_{F, C}$; and then the interference causes from CCI (co-channel interference) is with $I_{C, F}$, which is able to be ignored under the condition that the distance between fetmocell and macrocell site is assumed as large enough [17].

\section{The BER and OP Analysis}

To obtain the BER and OP of the system, the calculation of SINR for the signal at the output of decision maker is necessary. It is not only for determining the system average BER but for obtaining the outage probability experienced by macrocell and femtocell as subcarrier is below a threshold, i.e., the femtocel/macrocell outage probability is used to defined as the probability that the SINR, $\left(E_{b} / N_{0}\right)_{f}$ and $\left(E_{b} / N_{0}\right)_{m}$, of the corresponding system is lower than a pre-defined value. Generally, small coverage size of a femtocell is considered since the tier of femtocell is installed in indoor application. Based on such assumption, the variance of $I_{F, F}$ is able to be written as [12]

$$
\sigma_{I_{F, F}}^{2}=\left(Q_{F} \cdot \bar{\Psi}_{i}\right) /\left|\mathrm{X}_{i}\right|^{\alpha}
$$

where

$$
Q_{F}=P_{r, F} \cdot R_{f}^{\beta} \cdot\left(\frac{\lambda_{M}}{\lambda_{F}}\right) \frac{\left(d_{0, M}\right)^{\alpha}}{\left(d_{0, F}\right)^{\beta}}
$$

and

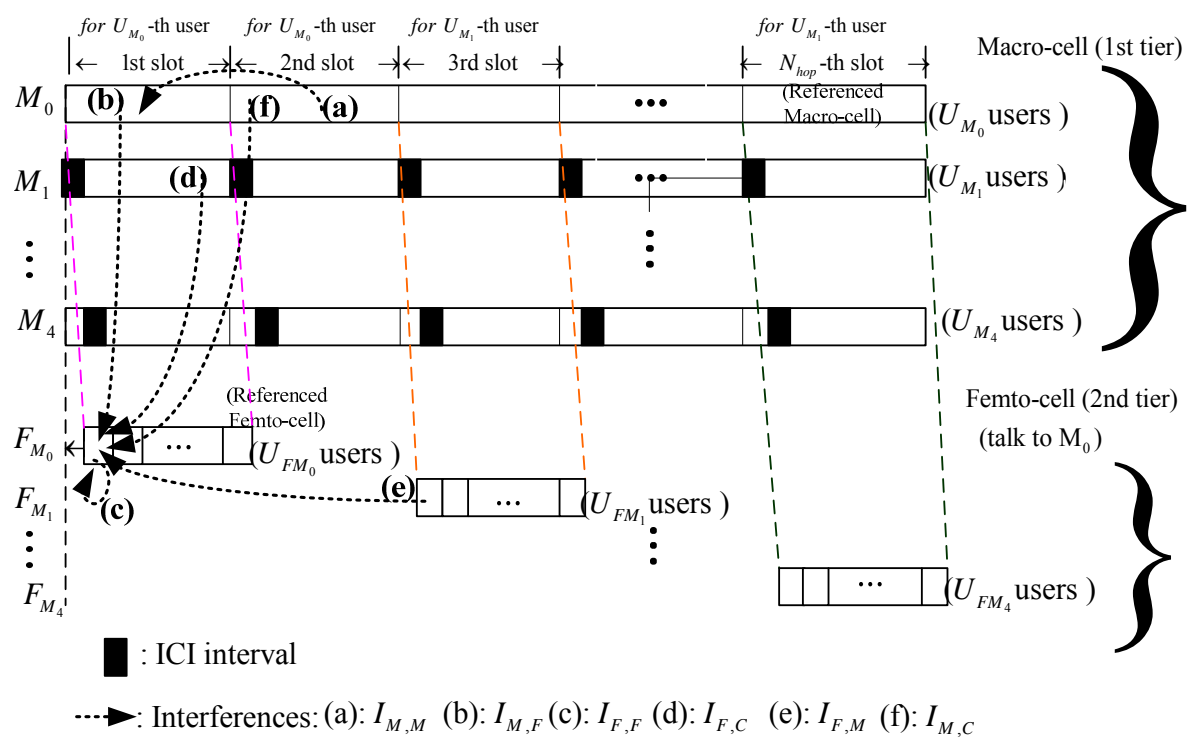

Figure 3. The structure of interferences within two-tier network. 


$$
\bar{\Psi}_{i}=\sum_{k=1}^{K} \frac{Q_{k, F_{j}}}{Q_{k, F_{i}}}
$$

The same second moment for $Q_{l, F_{j}}$ and $Q_{l, F_{i}}$ is represented as $\sigma_{d B}^{2}$, and $\bar{\Psi}_{i}$ is a log-normal distribution with variance $2 \sigma_{d B}^{2}$. The $\lambda_{M}$ and $\lambda_{F}$ are two empirical parameters designated as $\lambda_{M}=\left[C / 4 \pi f_{C} d_{0 M}\right]^{2}$ and $\lambda_{F}=\left[C / 4 \pi f_{c} d_{0 F}\right]^{2}$, respectively, where $d_{0 M}$ and $d_{0 F}$ are corresponding to the referenced distance for indoor loss of femtocell and macrocell, and $C=3 \times 10^{8}$ $\mathrm{m} / \mathrm{s}, f_{c}$ is the Doppler frequency. By substituting (7) and (8) into (2), the variance of $I_{F, F}$ can be obtained easily as determined as ceived power of a macrocell user.

$$
\begin{gathered}
\sigma_{I_{F, F}}^{2}=\left[P_{r, F} R_{f}^{\beta}\left(\frac{\lambda_{M}}{\lambda_{F}}\right) \frac{\left(d_{0, M}\right)^{\alpha}}{\left(d_{0, F}\right)^{\beta}}\right] /\left|X_{i}\right|^{\alpha} \quad \text { (9) } \begin{array}{l}
\text { after substituting all of the second } \\
\text { the statistically evaluated equation } \\
\text { SINR formula, and which is obtained }
\end{array} \\
\gamma_{S I N R}=\frac{S / 2}{\sum_{i=\Pi_{M}} 2 \sigma_{d B}^{2}\left(\frac{\left|X_{i}\right|}{\left|Y_{i}\right|}\right)^{\alpha}+0.2 \sigma_{d B}^{2} R_{f}^{\beta}\left(\frac{\lambda_{M}}{\lambda_{F}}\right)\left(\frac{d_{0 M}^{\alpha}}{d_{0 F}^{\beta}}\right) \cdot U_{f}\left|X_{i}\right|^{-\alpha}+0.1\left(U_{f}-1\right) \sigma_{d B}^{2}+\frac{N_{0}}{4 E_{b}}}
\end{gathered}
$$

Next, consider there are $U_{f}$ activating users are working in the femtocell, the variance of $I_{F, I}$ can be

$$
\sigma_{I_{F, I}}^{2}=\left(U_{f}-1\right) P_{r,(m)}
$$

Finally, the variance of $I_{F, C}$ is able to be evaluated as

$$
\sigma_{I_{F, C}}^{2}=\sum_{i=\Pi_{c}} P_{r, c} \bar{\Psi}_{i}(|\mathrm{X}| /|Y|)^{2}
$$

where $\bar{\Psi}_{i}$ has been defined in (8), and $P_{r, c}$ is the re-

It is easy to obtain the SINR expression of the system after substituting all of the second moment results from the statistically evaluated equations into the specified SINR formula, and which is obtained as (see Appendix)

$Q$-function, which can be alternate expressed as [14]

where $S=\sum_{n=0}^{N-1}\left(\beta_{n, o}^{2}\right)$.

$$
Q(t)=\frac{1}{\pi} \int_{0}^{\frac{\pi}{2}} \exp \left(\frac{-t^{2}}{2 \sin ^{2} \theta}\right) \mathrm{d} \theta
$$

Once the SINR is obtained the evaluation of system performance with the BER, consequently, with coherent demodulation technique the BER conditioned on the instantaneous SINR for an MC-CDMA system working in an two-tier femtocell cellular network is given as [14]

$$
P_{r}\left(\operatorname{error} \mid \beta_{l}, l=0,1, \cdots, L-1\right)=Q(\sqrt{\operatorname{SINR}})
$$

where $\beta_{l}, l=0,1, \cdots, L-1$, present the mean value of the desired signal, and $Q(\cdot)$ is the well-known Macuamm

$$
P_{a v}=\overbrace{\int_{0}^{\infty} \int_{0}^{\infty} \cdots \int_{0}^{\infty}}^{L} P_{r}\left(\text { error } \mid \alpha_{l}, l=0,1, \cdots, L-1\right) \times f_{\alpha_{0}, \cdots, \alpha_{(L-1)}}\left(\alpha_{0}, \cdots, \alpha_{(L-1)}\right) \cdot \mathrm{d} \alpha_{0} \mathrm{~d} \alpha_{1} \cdots \mathrm{d} \alpha_{L-1}
$$

The previous equation involving $L$-fold integration can be evaluated by the detailed means given in [18].

Moreover, another criterion for evaluating the system performance is to evaluate the OP at some cell site where the events of femtocell BSs and the users deployed in the designated environment are assumed as Poisson random. It is defined the uplink capacity of a two tier network as the maximum number of including femtocell BS and macrocell subscribers can supply to cause the OP of the total users experience within a two tier system is going to down below a threshold value and that is determined as

$$
P_{O}=\operatorname{Pr}\left(\frac{\left(S_{F E M}\right)^{2}}{\sigma_{I_{T}}^{2}}<\gamma_{T}\right)
$$

where $\gamma_{T}$ represents a preset threshold value of SNR, and the equation illustrated previously conditioning on the number can be advanced obtained as

$$
P_{O}=\frac{\exp \left(-E\left[I_{T}\right]\right)}{1-\exp \left(-E\left[I_{T}\right]\right)} \sum_{n=1}^{\infty} \frac{\left(E\left[I_{T}\right]\right)^{n}}{2 n !} \operatorname{erfc}\left(\frac{\frac{N}{\Gamma_{x}}-(n-1)-\left(E\left[I_{T}\right] \sigma_{I_{T}}^{2}\right)}{\sqrt{2 E\left[I_{T}\right] \sigma_{I_{T}}^{2}}}\right)
$$

where $E[\cdot]$ is a expect operator, $N$ indicts the processing gain, $\operatorname{erfc}(\cdot)$ denotes the complementary error function, and $\Gamma_{x}$ is the BER of the corresponding BS, that is, $\Gamma_{x}=\left\{\begin{array}{l}\left(E_{b} / N_{0}\right)_{f}, x=\text { femtocell } B S \\ \left(E_{b} / N_{0}\right)_{m}, x=\text { macrocell } B S\end{array}\right.$. 


\section{Numerical Results and Discussion}

It seems natural to make sense that the hopping numbers of a TH-CDMA system generated to serves different femtocells surrounding around a macrocell definitely dominates the performance of a two-tier femtocell networks. On the basis of derived formulas the results for proving the facts aforementioned are illustrated in Figure 4. The processing gain is considered as, $N=128$, and the OP plots with different hopping number $N_{\text {hop }}=1,2$, and 3 . Now, it clearly proves that the OP is going to become superior while the hopping numbers provided by the TH-CDMA system is increase, i.e., assume that the case is with same OP value, 0.01, TH-CDMA system can offer the resources to about 20 users with 3hopping numbers, however, this is contrast to just about to 5 users that is with only one hopping number. This is proved by the results shown in Figure 5, in which the hopping number is with $N_{\text {hop }}=1$, and $N_{\text {hop }}=2$ are compared with different values of radius of marcocell, $d_{o c}=500,1000,2000,3000$ meters, the user number is set as $U_{f}=2$, and the subcarrier number is set as $N=16$, respectively. It is easy to understand that the more of the hopping numbers, the better of the system performance. In other words, the more of the hopping numbers can serve to larger number of femtocell users and the OP will become decrease. Certainly, the results show that the system performance becomes deterioration when the radius is growing gradually. With the parameter $d_{o c}=500$ meter, the comparison results from different hopping numbers, $N_{\text {hop }}=1,2$, and different subcarrier numbers, $N=8,16,32,64$, are shown in Figure 6, where the curves illustrate that the system performance becomes superior once the number of subcarrier is increase. Especially, it is worth noting that the phenomenon is much significant during the larger SNR $\left(E_{b} / N_{0}\right)$ value. This point is accordance with the general characteristics of an multi-carrier system. Assigning subcarrier number $N=16$ and distance between macrocell and femtocell $d_{o c}=2000$ meter in Figure 7, respectively. The pictorial shows the BER curves of an MC-CDMA system in a femtocell environment, $U_{f}=1,2,3$ and $N_{\text {hop }}=1,2,3$ are corresponding to adopt as different user numbers and different hopping numbers. It is obviously to discover that when the time-hopped CDMA system provides with fewer hopping number to an MC-CDMA system's user who is activating under one of the referenced femtocell, the inferior system performance is going to be obtained. This fact is supported again by the reason that the hopping number definitely dominates the system performance of a multi-subcarrier system operating within a two-tier femtocell network. However, there is one critical problem

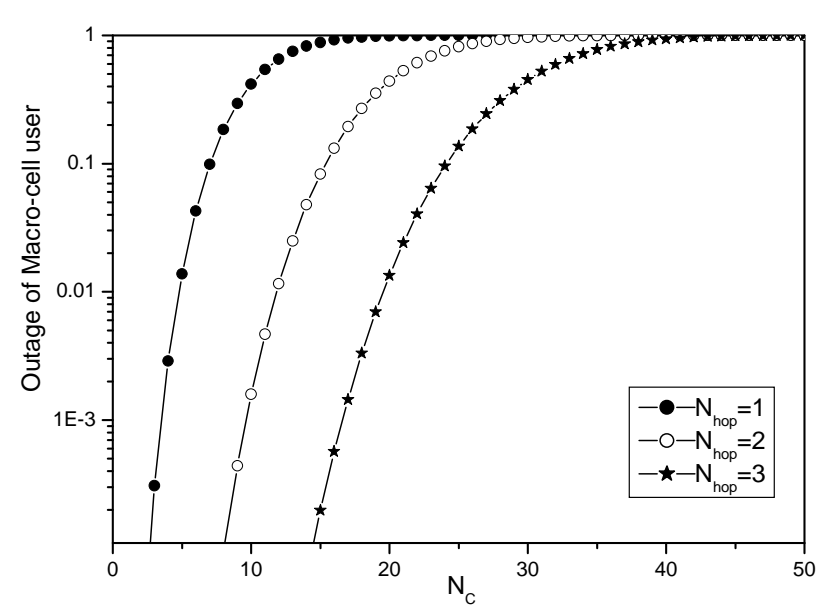

Figure 4. The macrocell user OP of a two tire network with different hopping numbers.

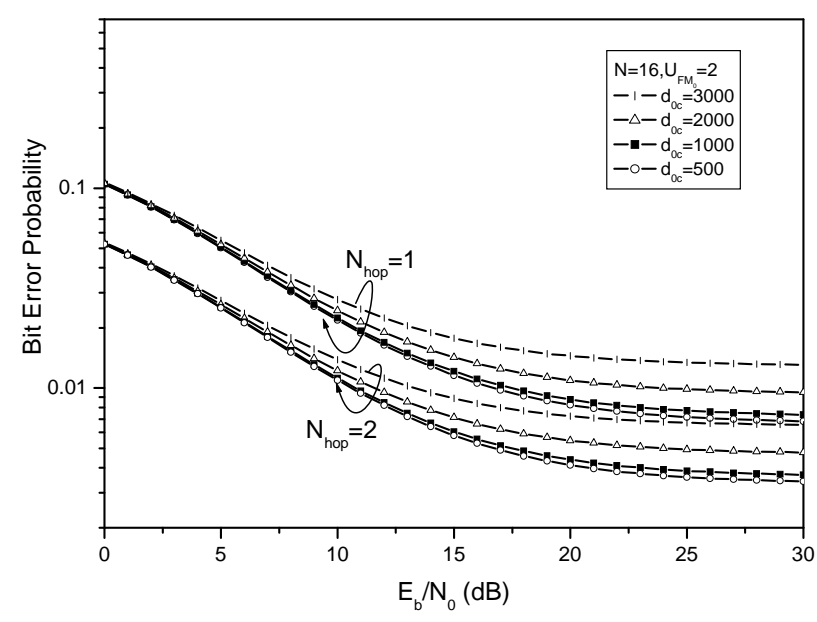

Figure 5. The plots of SNR $\left(E_{b} / N_{0}\right)$ vs BER under the assumption of different hopping numbers.

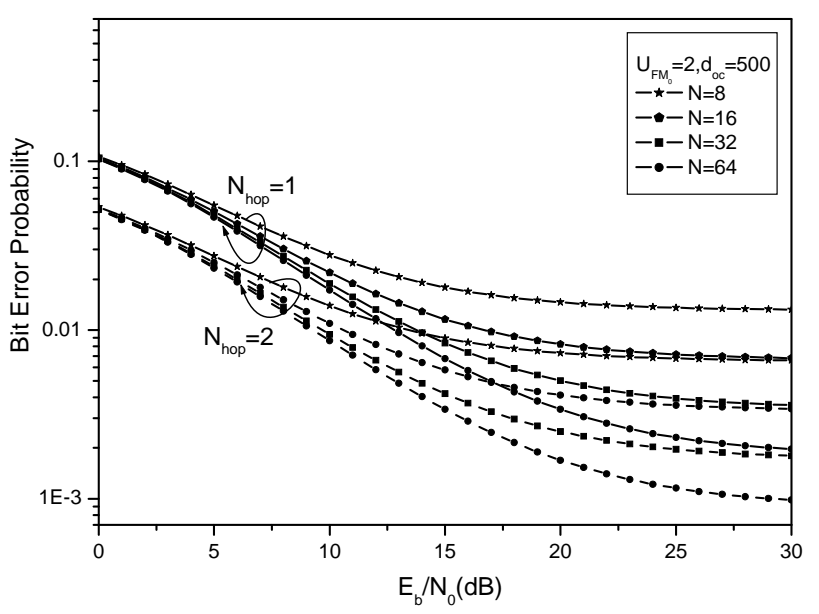

Figure 6. The plots of SNR $\left(E_{b} / N_{0}\right)$ vs BER under the assumption of different subcarrier numbers. 


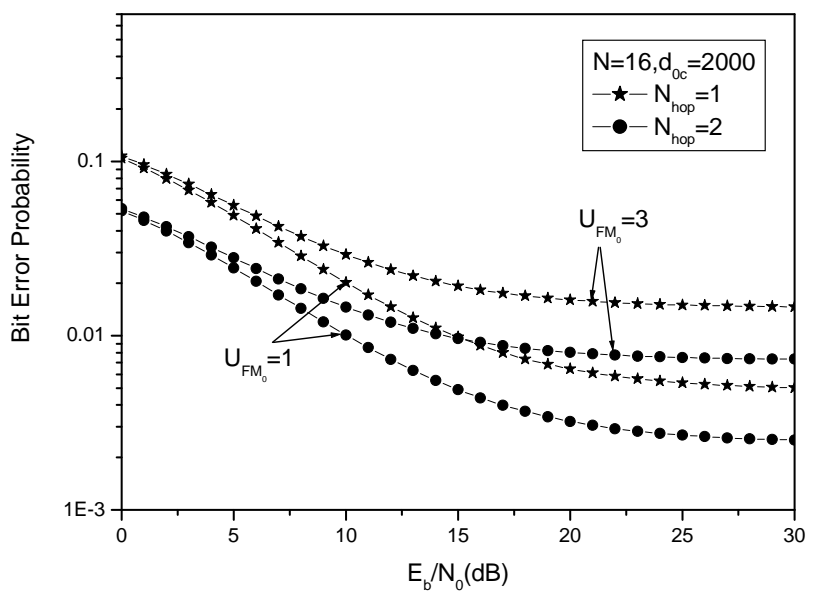

Figure 7. The plots of SNR $\left(E_{b} / N_{0}\right)$ vs BER under the assumption of different subscriber numbers and different hopping numbers.

exists in Figure 7 that the curve under the conditions of $U_{f}=1$ and $N_{\text {hop }}=1$ is becoming superior to the curve with conditions of $U_{f}=3$ and $N_{\text {hop }}=2$ after the BER is rising to about $E_{b} / N_{0}=15$. This describes that the system performance will trend to degradation when the number of user is increasing within an femtocell, the reason is the multiple access interference (MAI) getting larger gradually.

\section{Conclusion}

In the paper the evaluation of system performance for an MC-CDMA (multi-carrier coded-division multiple-access) system operating over single-cell with two-tier femtocell environment is derived and analyzed with the numerical results. The scenario is assumed that there are several femtocells arbitrarily distributed around a macrocell, and the Rayleigh fading environment is considered. Under the consideration of TH-CDMA scheme in alternatively spreading the data to each user with fair opportunity, the results show that the system performance is definitely dominated by the parameter of the hopping number. In addition, the number of subcarrier is still one of the important factors to affect the system performance of the MC-CDMA system operating in multi-user transmission systems.

\section{References}

[1] V. Chandrasekhar, J. G. Andrews and A. Gatherer, "Femtocell Networks: A Survey," IEEE Communication Magazine, Vol. 46, No. 9, September 2008, pp. 59-67. doi:10.1109/MCOM.2008.4623708

[2] S.-P. Yeh, S. Taluar, S.-C. Lee and H. Kim, "WiMAX Femtocells: A Perspective on Network. Architecture,
Capacity, and Coverage," IEEE Communication Magazine, Vol. 46, No. 10, October 2008, pp. 58-65. doi:10.1109/MCOM.2008.4644120

[3] T. E. Klein and S.-J Han, "Assignment Strategies for Mobile Data Users in Hierarchical Overlay Networks: Performance of Optimal and Adaptive Strategies," IEEE Journal on Selected Areas in Communications, Vol. 22, No. 5, June 2004, pp. 849-861. doi:10.1109/JSAC.2004.826922

[4] S. Kishore, L. J. Greenstein, H. V. Poor and S. C. Schwartz, "Soft Hand Off and Uplink Capacity in a Two-tier CDMA System," IEEE Transactions on Wireless Communications, Vol. 4, No. 4, July 2005, pp. 1297 1301. doi:10.1109/TWC.2005.850319

[5] V. Chandrasekhar, J. G. Andrews and A. Gatherer, "Uplink Capacity and Interference Avoidance for Two-Tier Femtocell Networks," IEEE Transactions on Wireless Communications, Vol. 8, No. 7, July 2009, pp.1-12. doi:10.1109/TWC.2009.070475

[6] V. Chandrasekhar, J. G. Andrews and A. Gatherer, "Coverage in Multi-Antenna Two-tier Networks," IEEE Transactions on Wireless Communications, Vol. 8, No. 10, October 2009, pp. 5314-5327. doi:10.1109/TWC.2009.090241

[7] H. C. Claussen, L. T. W. Ho and L. G. Samual, "Selfoptimization of Coverage for Femtocell Deployments," Wireless Telecomunications Symposium, April 2008, pp. 278-285. doi:10.1109/WTS.2008.4547576

[8] S. Kishore, L. J. Greenstein, H. V. Poor and S. C. Schwartz, "Soft-handoff and Uplink Capacity in a Two-tier CDMA System," IEEE Transactions on Wireless Communications, Vol. 4, No. 4, July 2005, pp. 12961301. doi:10.1109/TWC.2005.850319

[9] V. Chandrasekhar, J. G. Andrews and A. Gatherer, "Power Control in Two-tier Femtocell Networks," To Appear, IEEE Transactions on Wireless Communications, Vol. 8, No. 8, August 2009, pp. 4316-4328. doi:10.1109/TWC.2009.081386

[10] A. Ghasemi and E. Sousa, "Spectrum Sensing in Cognitive Radio Networks: The Cooperation-Processing Tradeoff," Wireless Communications and Mobile Computing, Vol. 7, No. 9, November 2007, pp. 1049-1060. doi: $10.1002 /$ wcm. 480

[11] L. Qian, X. Li, J. Attia and Z. Gajic, "Power Control for Cognitive Radio Ad hoc Networks," Proceedings of IEEE Workshop on Local and Metropolitan Area Networks, June 2007, pp. 7-12. doi:10.1109/LANMAN.2007.4295967

[12] A. M. Hunter, J. G. Andrews and S. Weber, "Transmission Capacity of Ad hoc Network with Spatial Diversity," IEEE Transactions on Communications, Vol. 7, No. 12, December 2008, pp. 5058-5071.

[13] L.-L. Yang, "Time-Hopping Multicarrier Code-Division Multiple Access," IEEE Tranactions on Vehicular Technology, Vol. 56, No. 2, March 2007, pp. 731-741. doi:10.1109/TVT.2006.889577

[14] D. L. Perez, A. Valcarce, G. de la Roche and J. Zhang, "OFDMA Femtocells: A Roadmap on Interference 
Avoidance," IEEE Communications Magazine, September 2009, pp. 41-48. doi:10.1109/MCOM.2009.5277454

[15] J. F. C. Kingman, "Poisson Processes," Oxford Science, Oxford, 1993.

[16] M. Haenggi, J. G. Andrew, F. Baccelli, O. Dousse and M. Franceschetti, "Stochastic Geometry and Random Graphs for the Analysis and Design of Wireless Networks," IEEE Journal on Selected Areas in Communications, Vol. 27, No. 7, September 2009, pp. 1029-1046. doi:10.1109/JSAC.2009.090902
[17] H.-S. Jo, C. Mun, J. Moon and J.-G. Yook, "Interference Mitigation Using Uplink Power Control for Two-Tier Femtocell Networks," IEEE Transactions on Wireless Communications, Vol. 8, No. 10, October 2009, pp. 4906-4910. doi:10.1109/TWC.2009.080457

[18] M. K. Simon and M. S. Alouini, "A Unified Approach to the Performance Analysis of Digital Communication over Generalized Fading Channel," Proc. of the IEEE, Vol. 86, No. 9, September 1998, pp. 1860-1877. doi:10.1109/5.705532

\section{Appendix}

The results of SINR for a referenced user working in an MC-CDMA system located at femtocell within two-tier system can be obtained by substituting the average desired signal and the summation of all the interferences shown previous, and calculated as

$$
\begin{aligned}
& \gamma_{S I N R}=\frac{\left[\sqrt{\frac{P_{r, F}}{2}} \sum_{n=0}^{N-1}\left(\beta_{n, o}\right)^{2}\right]^{2}}{I_{F, M}+I_{F, F}+I_{F, C}+I_{A W G N}} \\
& =\frac{\frac{P_{r, F}}{2}\left(\sum_{n=0}^{N-1}\left(\beta_{n, o}^{2}\right)\right)^{2}}{\left(\sum_{i=\Pi_{M}} P_{r, M} \cdot 2 \sigma_{d B}^{2}\left(\frac{\left|\mathrm{X}_{i}\right|}{\left|Y_{i}\right|}\right)^{2}+P_{r, F} 2 \sigma_{d B}^{2} R_{f}^{\beta}\left(\frac{\lambda_{c}}{\lambda_{f}}\right)\left(\frac{d_{0 M}^{\alpha}}{d_{0 F}^{\beta}}\right) \cdot U_{F M_{0}}\left|\mathrm{X}_{i}\right|^{-\alpha}+\left(U_{F M_{0}}-1\right) P_{r, F}+\frac{N_{0}}{4 T_{b}}\right) \sum_{n=0}^{N-1}\left(\beta_{n, o}^{2}\right)}
\end{aligned}
$$

where the received average bit power of the users within macrocell, $\mathrm{P}_{r, M}$, is assumed as ten multiplied by the power of the users within femtocell, $\mathrm{P}_{r, F}$, i.e.,
$P_{r, M}=10 P_{r, F}, \Pi_{M}$ represents the chosen macrocell, and let $s=\sum_{n=0}^{N-1}\left(\beta_{n, o}^{2}\right)$. Thus, the $\gamma_{\text {SINR }}$ becomes as

$$
\gamma_{S I N R}=\frac{\frac{P_{r, M}}{2} s^{2}}{\left(\sum_{i=\Pi_{M}} P_{r, M} \cdot 2 \sigma_{d B}^{2}\left(\frac{\left|\mathrm{X}_{i}\right|}{\left|Y_{i}\right|}\right)^{\alpha}+0.1 P_{r, F} 2 \sigma_{d B}^{2} R_{f}^{\beta}\left(\frac{\lambda_{M}}{\lambda_{M}}\right)\left(\frac{d_{0 M}^{\alpha}}{d_{0 F}^{\beta}}\right) \cdot U_{F M_{0}}\left|\mathrm{X}_{i}\right|^{-\alpha}+\left(U_{F M_{0}}-1\right) 0.1 P_{r, F} \sigma_{d B}^{2}+\frac{N_{0}}{4 T_{b}}\right) s}
$$

By cancelling the parameter $\mathrm{s}$, and substituting $E_{b}=T_{b} \cdot P_{r, F}$ into the previous equation, it becomes as

$$
\gamma_{S I N R}=\frac{s / 2}{\left(\sum_{i=\Pi_{M}} 2 \sigma_{d B}^{2}\left(\frac{\left|\mathrm{X}_{i}\right|}{\left|Y_{i}\right|}\right)^{\alpha}+0.2 \sigma_{d B}^{2} R_{f}^{\beta}\left(\frac{\lambda_{M}}{\lambda_{F}}\right)\left(\frac{d_{0 M}^{\alpha}}{d_{0 F}^{\beta}}\right) \cdot U_{F M_{0}} \cdot\left|\mathrm{X}_{i}\right|^{-\alpha}+0.1\left(U_{F M_{0}}-1\right) \sigma_{d B}^{2}+\frac{N_{0}}{4 E_{b}}\right)}
$$

